\title{
Die Vorschläge der FMH zum zukünftigen Gesundheitswesen
}

Anlässlich einer Anhörung durch die Kommission für soziale Sicherheit und Gesundheit des Ständerats hatte eine Delegation der FMH und der Schweizerischen Konferenz der kantonalen Gesundheitsdirektorinnen und -direktoren (GDK) kürzlich die Gelegenheit, ihre Vorschläge für die Zukunft unseres Gesundheitssystems zu präsentieren.

Lassen Sie mich im folgenden auf einige der Punkte eingehen, die an diesem Treffen behandelt wurden.

Im Rahmen unserer Delegiertenversammlung haben wir bereits vor mehreren Monaten festgehalten, dass das Gesundheitswesen einer Steuerung bedarf; ohne eine Mindestmass an Steuerung besteht die Gefahr, dass das Gesundheitssystem in ein Chaos ausartet und dies anschliessend sehr viel einengendere Planungsmassnahmen zur Folge hat.

Was diese Steuerung anbelangt, ist es für uns wesentlich vorteilhafter, wenn diese Verantwortung von den politischen Behörden - mit Beteiligung der Ärztinnen und Ärzte als Experten - wahrgenommen wird, statt dass man diese Aufgabe den Krankenkassen überlässt, die eine andere Funktion zu erfüllen haben.

Diesbezüglich sei an das Abstimmungsergebnis vom 1. Juni erinnert, mit dem das Stimmvolk sehr klar zum Ausdruck brachte, dass sich die Krankenkassen auf ihre Kernaufgabe beschränken und ihre Kompetenzen nicht überschreiten sollen.

In diesem Zusammenhang müssen wir klar zwischen zwei Ebenen unterscheiden:

- zum einen die Ebene der politischen Verantwortung dabei geht es um die Definition der Rahmenbedingungen des Gesundheitssystems und der Kontrollgrundsätze sowie um die Festlegung des «Gesamtvolumens des Systems», einschliesslich der Fragen im Bereich der Ärztedemographie; diesbezüglich kann die Ärzteschaft ihr Know-how einbringen;

- zum anderen die Ebene des Betriebs dieses Systems innerhalb des festgelegten Rahmens - dabei geht es beispielsweise um die Tarife, die Modalitäten von Managed Care, die Qualitätskriterien usw.; auf dieser zweiten Ebene besteht die Aufgabe der Ärzteschaft darin, dass sie in den Verhandlungen als Partner agiert.
Eine klare und permanente Trennung zwischen diesen beiden Kompetenzebenen ist unserer Meinung nach unerlässlich: Die Bestimmung des Rahmens des Gesundheitssystems ist Sache der Politik, die ihre Entscheide gegenüber den Wählerinnen und Wählern zu verantworten hat. Dies ist nicht die Aufgabe der Versicherer, deren Berechtigung in einem anderen Bereich liegt.

Wenn eine klare Trennung zwischen diesen beiden Ebenen vorgenommen wird (zum einen politische Festlegung der Rahmenbedingungen des Gesundheitssystems, zum anderen Steuerung des Systems), kann verhindert werden, dass beispielsweise die Tarifverhandlungen, die Gespräche über Managed Care oder die Überlegungen über die Qualitätskontrollen durch eine übertriebene Ausrichtung auf die Steuerung des Systems beeinträchtigt werden.

Gegenwärtig wird über Qualitätskriterien diskutiert, wobei es in Wirklichkeit um eine Begrenzung der Zahl der zugelassenen Ärztinnen und Ärzte geht. Man spricht von Managed Care, um einen Zustrom von ausländischen Ärztinnen und Ärzten zu verhindern, doch das ist der falsche Ansatz!

Wenn man jedoch diese beiden Kompetenzebenen klar voneinander trennt, wenn man klar festhält, dass die partnerschaftlichen Verhandlungen mit den Versicherern nicht mehr die Festlegung des Systems, sondern dessen Steuerung zum Ziel haben, dann geht es bei den Gesprächen über Managed Care tatsächlich um Managed Care und bei den Diskussionen über die Qualitätskriterien wirklich um die Qualität. Unter solchen Voraussetzungen sind die Wirtschaftlichkeitsprüfungen glaubwürdig, da die Zahl der Ärztinnen und Ärzte über die Rahmenbedingungen des Systems gesteuert wird.

Wir freuen uns somit über die Tatsache, dass künftig jeder seine Verantwortung auf seiner Ebene wahrnimmt dies wird sicherlich konstruktivere und offenere Diskussionen sowie eine bessere Berücksichtigung der Kompetenzen und der Position aller Beteiligten ermöglichen.

Dr. med. Jacques de Haller, Präsident der FMH 